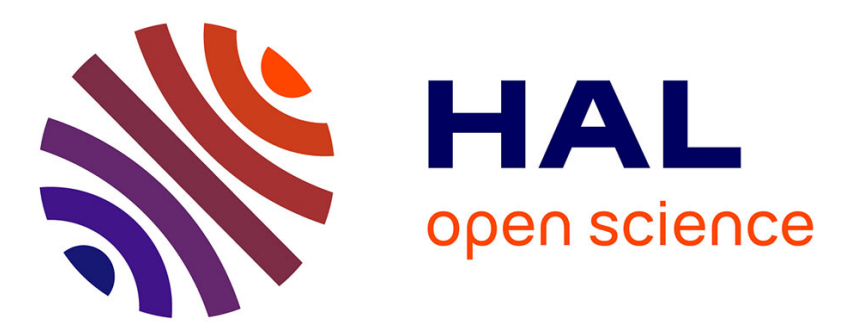

\title{
Affichage d'informations par des impulsions haptiques
}

\author{
Thomas Pietrzak, Benoît Martin, Isabelle Pecci
}

\section{To cite this version:}

Thomas Pietrzak, Benoît Martin, Isabelle Pecci. Affichage d'informations par des impulsions haptiques. Proceedings of the AFIHM Conférence Francophone sur l'interaction Homme-Machine (IHM 2005), 2005, Toulouse, France. hal-01821167

\section{HAL Id: hal-01821167 \\ https://hal.inria.fr/hal-01821167}

Submitted on 22 Jun 2018

HAL is a multi-disciplinary open access archive for the deposit and dissemination of scientific research documents, whether they are published or not. The documents may come from teaching and research institutions in France or abroad, or from public or private research centers.
L'archive ouverte pluridisciplinaire HAL, est destinée au dépôt et à la diffusion de documents scientifiques de niveau recherche, publiés ou non, émanant des établissements d'enseignement et de recherche français ou étrangers, des laboratoires publics ou privés. 


\title{
Affichage d'informations par des impulsions haptiques
}

\author{
Thomas Pietrzak - Benoît Martin - Isabelle Pecci \\ Université Paul Verlaine - Metz, LITA \\ UFR MIM, île du Saulcy \\ 57045, Metz Cedex 1, France \\ \{pietrzak, benoit.martin, pecci\}@univ-metz.fr
}

\begin{abstract}
RESUME
Notre but était de trouver des effets haptiques utilisables pour présenter des informations à l'utilisateur d'un périphérique haptique. Nous présentons une étude sur la discrimination d'impulsions haptiques effectuées par un périphérique à retour d'effort de type PHANToM. Les paramètres testés ici sont la direction et l'amplitude des impulsions. Les résultats montrent que la discrimination de direction est aisée alors que la discrimination d'amplitude n'est pas triviale. Seulement deux niveaux d'amplitude peuvent être distingués de manière exacte.
\end{abstract}

MOTS CLES : Retour d'effort, affichage d'informations, multi modalité.

\section{ABSTRACT}

Our goal was to find haptic effects that could be used to present information to the user of a haptic pointing device. We present a study on users' ability to discriminate between different effects presented with a PHANToM haptic pointing device. The effects we experimented with were bumps that the user could feel through the PHANToM. The direction and the amplitude of the bumps were manipulated. The results show that the direction is easy to discriminate, but the amplitude is not. Only two levels of amplitude could be reliably discriminated.

CATEGORIES AND SUBJECT DESCRIPTORS: H.5.2 [User Interfaces]: Haptic I/O; H.1.2 [User/Machine Systems]: Human information processing.

GENERAL TERMS: Human Factors, Measurements.

KEYWORDS: Force feedback, information display, multi-modality.

\section{INTRODUCTION}

La recherche décrite dans cet article concerne l'affichage d'informations. Cela peut se faire par plusieurs canaux,

Permission to make digital or hard copies of all or part of this work for personal or classroom use is granted without fee provided that copies are not made or distributed for profit or commercial advantage and that copies bear this notice and the full citation on the first page. To copy otherwise, or republish, to post on servers or to redistribute to lists, requires prior specific permission and/or a fee. IHM 2005, September 27-30, 2005 Toulouse, France. Copyright 2005 ACM X-XXXXX-XXXX/XXIXXXX $\$ 5.00$ soit pour proposer à l'utilisateur de choisir le canal qui lui convient, soit pour en utiliser plusieurs en même temps, soit pour éviter de surcharger un canal déjà très utilisé. Ceci permet notamment de remplacer un canal déficient. Les canaux visuels et auditifs sont très utilisés alors que d'autres canaux comme l'haptique qui regroupe le sens tactile et celui du retour d'effort, restent peu répandus. Des études sur l'affichage d'informations auditives ont été faites. On peut notamment citer les auditory icons de Gaver [4] : ce sont des métaphores sonores qui permettent d'associer un son à un objet ou une action. Par exemple la suppression d'un fichier peut être signalée par le son du froissement d'une feuille de papier. L'inconvénient est que les icônes sont choisies arbitrairement et que la compréhension de l'information passe par des connaissances pragmatiques partagées entre le créateur de l'icône et l'utilisateur.

Un autre système a été imaginé par Brewster [3] : il s'agit des earcons. Là ce ne sont plus des sons qui sont utilisés mais des notes. Des codes de plusieurs niveaux sont créés pour afficher des informations hiérarchiques. Au premier niveau seul le rythme change, chaque rythme correspondant à une catégorie. Pour différencier des codes d'une même catégorie Brewster crée des informations d'un niveau supérieur en modifiant la mélodie. Au niveau suivant c'est le tempo global qui change. Ainsi on peut faire un lien entre plusieurs informations en comparant le rythme, la mélodie et le tempo. Cependant comme le canal auditif n'est pas toujours approprié (par exemple dans le cas des malentendants et en milieu bruité), Brewster et Brown [2] ont étendu leur approche aux icônes tactiles appelées tactons. L'idée est de créer des vibrations en faisant varier le rythme, la fréquence et la durée. Le principe est le même que pour les earcons : utiliser trois paramètres pour hiérarchiser les codes. Nous cherchons à savoir si ce principe est extensible au retour d'effort et avec quels paramètres.

MacLean et Enriquez [5] ont fait une étude sur des icônes haptiques en utilisant le retour d'effort. Ils utilisent un moteur à courant continu qui délivre des forces sur un axe en rotation : le signal envoyé peut se paramétrer en magnitude (amplitude de déplacement), en forme (signal sinusoïdal, carré, etc.) et en fréquence. Les études publiées montrent des tests utilisateurs où les participants devaient classer des icônes utilisant ces paramètres en catégories de leur choix. Il semble que le seul critère 
unanimement utilisé pour la classification est la fréquence.

Actuellement l'affichage de messages par le retour d'effort est très peu étudié, c'est ce à quoi nous nous intéressons dans le projet PICOB (Post-It haptiques par COdesBarres), lui même intégré dans le projet européen MICOLE auquel nous participons. Ce projet a pour but de créer un environnement d'apprentissage collaboratif multimodal pour déficients visuels.

\section{AFFICHAGE PAR LE RETOUR D'EFFORT}

Pour afficher un message il faut d'abord trouver un moyen de le coder puis un moyen de représenter le codage. Un exemple bien connu de codage est le code morse : il utilise deux digits pour coder les caractères alphanumériques. Pour afficher un message utilisant ce code il faut associer une représentation à chaque digit. Une représentation auditive courante est des bips longs et courts, et au niveau visuel des tirets et des points. Pour afficher le message " SOS» avec le codage morse il faut utiliser une de ses représentations. On obtient par

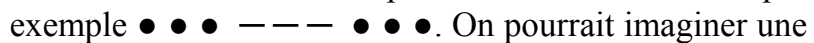
représentation haptique, par exemple avec des impulsions vers le haut et vers le bas. Le codage utilisé serait le même mais le message serait alors $\downarrow \downarrow \downarrow \uparrow \uparrow \uparrow \downarrow \downarrow \downarrow$.

Nous en sommes au stade préliminaire de ces recherches et parmi les types de représentations possibles que nous avons imaginés nous présentons ici les impulsions. Le principe est simple : il s'agit de donner des impulsions à la main de l'utilisateur sur quelques millimètres. Une impulsion est déterminée par trois paramètres : la durée, l'amplitude et la direction. La figure 1 représente des impulsions dans les six directions : haut, droite, arrière, bas, gauche et avant. Pour un périphérique à deux degrés de libertés on devra se limiter à quatre directions.
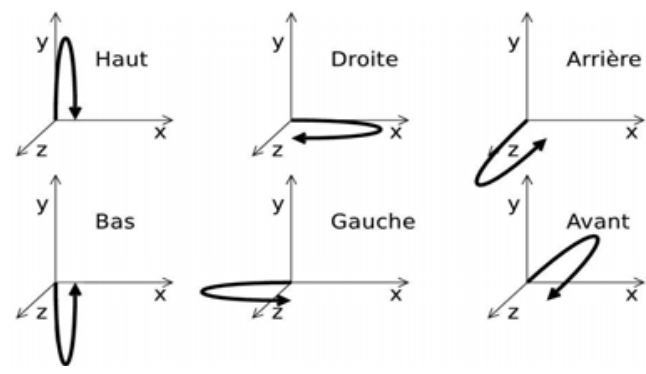

Figure 1 : Impulsions dans six directions différentes.

Le principe utilisé pour réaliser ces impulsions est simple : l'idée est de tirer le curseur ( $O$ sur la figure 2 ) avec un ressort exerçant une force $\mathrm{F}$ vers une position théorique de l'impulsion (• sur la figure 2 ).

\section{EXPERIMENTATIONS}

Les expériences ci-dessous ont pour but d'étudier la discrimination d'impulsions de directions et d'amplitudes différentes. Ces tests n'ont pas pour but d'étudier un apprentissage mais une utilisabilité immédiate.
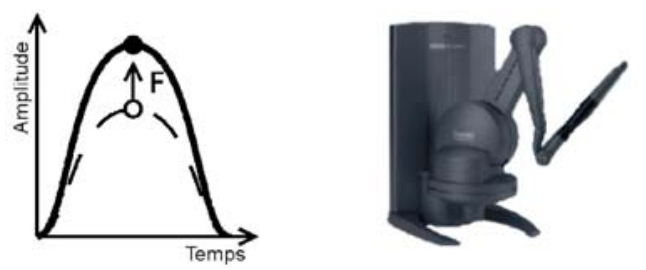

Figure 2 : Principe et périphérique utilisés pour donner une impulsion à l'utilisateur.

Il s'agit de faire ressentir des séries de 150 impulsions de $250 \mathrm{~ms}$ à des utilisateurs. Ils doivent déterminer la direction ou l'amplitude selon l'expérience. La durée des impulsions étant fixe, la discrimination selon ce paramètre n'a pas été testée : ce sera le sujet d'une étude ultérieure. La durée et les amplitudes ont été choisies lors de tests préliminaires afin d'avoir des impulsions a priori confortables à utiliser.

\section{Déroulement.}

Après avoir expliqué le principe de l'expérience à l'utilisateur, l'expérimentateur déclenche les impulsions une à une. L'utilisateur annonce la direction (haut, bas, gauche, droite, avant ou arrière) ou l'amplitude (1,2 ou 3) qu'il a ressenti et un quart de seconde plus tard l'impulsion suivante est déclenchée. Les participants étaient occultés afin de supprimer l'aide visuelle. Chaque expérimentation consistait à faire une ou plusieurs séries tirées aléatoirement et les utilisateurs faisaient tous les mêmes séries afin de n'avantager personne a priori. Les séries étaient regroupées en sessions, sachant que tous les utilisateurs faisaient les mêmes sessions au rythme de une par jour maximum. Après chaque session un questionnaire était distribué pour recueillir les impressions des participants.

\section{Matériel utilisé.}

Cette expérience a été réalisée avec un PHANToM Desktop [6] (figure 2) comme périphérique de pointage à retour d'effort. C'est un périphérique à six degrés de liberté (trois en translation et trois en rotation) et à retour d'effort sur les trois axes de translation. Le programme utilisé a été réalisé avec l'API Reachin [1] en utilisant VRML et Python.

\section{Participants.}

Six utilisateurs ont participé aux tests : trois enseignantschercheurs (deux hommes et une femme) et trois étudiants de sexe masculin. Les enseignants et un des étudiants sont habitués à manipuler le PHANToM, tous sont droitiers. Les utilisateurs avaient entre 23 et 47 ans.

\section{Expérience 1}

Expérimentations et procédure. Dans la première expérience il s'agit de tester la discrimination de la direc- 
tion. Les impulsions possibles étaient de six directions : haut, droite, arrière, bas, gauche et avant (figure 1). Cinq séries ont été faites, chacune utilisant une amplitude différente des autres : $0,4 \mathrm{~cm}, 0,7 \mathrm{~cm}, 1 \mathrm{~cm}, 1,5 \mathrm{~cm}$ et $2,25 \mathrm{~cm}$.

Résultats et discussions. Les erreurs sont reportées dans la table 1 : chaque ligne correspond à une série dont l'amplitude testée est précisée dans la première colonne. Le pourcentage d'erreurs indiqué dans la dernière colonne représente le pourcentage d'erreurs sur le total de tous les utilisateurs pour la série considérée.

\begin{tabular}{|c|c|c|c|c|c|c|c|}
\hline $\begin{array}{c}\text { Amplitude } \\
(\mathbf{c m})\end{array}$ & $\mathbf{1}$ & $\mathbf{2}$ & $\mathbf{3}$ & $\mathbf{4}$ & $\mathbf{5}$ & $\mathbf{6}$ & $\mathbf{( \% )}$ \\
\hline 0,4 & 0 & 0 & 0 & 0 & 1 & 1 & $0,22 \%$ \\
0,7 & 0 & 0 & 0 & 0 & 0 & 0 & $0,00 \%$ \\
1 & 0 & 0 & 0 & 0 & 0 & 0 & $0,00 \%$ \\
1,5 & 0 & 0 & 1 & 0 & 0 & 0 & $0,11 \%$ \\
2,25 & 0 & 1 & 0 & 0 & 0 & 0 & $0,11 \%$ \\
\hline Total & 0 & 1 & 1 & 0 & 1 & 1 & $0,09 \%$ \\
\hline
\end{tabular}

Table 1 : Expérience 1 : erreurs en direction

Deux utilisateurs n'ont fait aucune faute et les quatre autres ont fait une erreur sur les $5 \times 150$ impulsions. Le nombre d'erreurs est clairement négligeable, donc on peut affirmer que dans ces conditions on peut discriminer des impulsions de six directions différentes. Un seul utilisateur (non habitué au PHANToM) avoue avoir eu parfois des difficultés à sentir la différence entre les directions et trois autres disent avoir eu parfois des hésitations. Deux utilisateurs avaient le sentiment d'avoir fait plus de fautes qu'ils n'en ont fait en réalité. Deux autres utilisateurs disent avoir eu l'impression que l'amplitude n'était pas la même dans des directions différentes : un utilisateur a eu l'impression que la droite était plus grande que haut et bas, alors qu'un autre utilisateur a eu l'impression que haut, bas, gauche et droit était plus grand que avant et arrière. Notons que l'amplitude de $2,25 \mathrm{~cm}$ a été jugée trop violente par les utilisateurs, c'est pourquoi elle ne fut plus utilisée dans les expériences suivantes. Les grandes impulsions posent un problème de prise en main du stylet : il faut le maintenir plus fort.

\section{Expérience 2}

Expérimentations et procédure. Cette expérience a pour but de tester la discrimination d'impulsion de trois amplitudes différentes. Les impulsions de cette expérience étaient toutes dirigées vers le haut, et les amplitudes étaient de $0,4 \mathrm{~cm}, 0,95 \mathrm{~cm}$ et $1,5 \mathrm{~cm}$. Une seule série a été menée.

Résultats et discussion. Les erreurs sont reportées dans la table 2. Les trois premières lignes représentent les erreurs par amplitude proposée et la dernière représente le total sur la série. La dernière colonne représente le pourcentage de réponses erronées par amplitude proposée sur le total des utilisateurs. Les erreurs sont plus importantes que dans l'expérience précédente. Les utili- sateurs ont fait entre 4 et 15 erreurs sur les 150 impulsions. En moyenne il y a $6 \%$ d'erreurs, mais le point intéressant est que seulement $1 \%$ des impulsions de $0,4 \mathrm{~cm}$ étaient mal reconnues alors que pour les deux autres amplitudes on obtient $7 \%$ et $11 \%$. De plus pour les erreurs de discrimination de l'amplitude moyenne, les utilisateurs ont toujours répondu en faveur de la grande amplitude. Pour les autres erreurs, sur des petites et grandes amplitudes, les réponses des utilisateurs étaient en faveur de l'amplitude moyenne. Ainsi les utilisateurs ne se sont jamais trompés en répondant «petite amplitude». Il y a donc clairement un problème de discrimination entre l'amplitude moyenne et la grande amplitude. Ce phénomène se reproduit dans l'expérience 3 .

\begin{tabular}{|c|c|c|c|c|c|c|c|}
\hline Amplitude & \multicolumn{9}{|c|}{ Utilisateur } & Erreurs \\
$(\mathbf{c m})$ & $\mathbf{1}$ & $\mathbf{2}$ & $\mathbf{3}$ & $\mathbf{4}$ & $\mathbf{5}$ & $\mathbf{6}$ & $(\%)$ \\
\hline 0,4 & 0 & 1 & 0 & 0 & 1 & 1 & $1 \%$ \\
0,95 & 3 & 2 & 4 & 5 & 2 & 6 & $7 \%$ \\
1,5 & 3 & 10 & 7 & 10 & 1 & 2 & $11 \%$ \\
\hline Total & 6 & 13 & 11 & 15 & 4 & 9 & $6 \%$ \\
\hline
\end{tabular}

Table 2 : Expérience 2 : erreurs en amplitude

\section{Expérience 3}

Expérimentations et procédure. Ce que nous voulons tester dans cette expérience est la discrimination simultanée de la direction et de l'amplitude. Pour cela nous proposons des impulsions dans les six directions avec deux puis trois amplitudes. Trois séries ont été réalisées : dans la première il n'y avait que deux amplitudes $(0,4 \mathrm{~cm}$ et $1,6 \mathrm{~cm})$. Dans la deuxième il y en avait trois : $0,4 \mathrm{~cm}$, $0,95 \mathrm{~cm}$ et $1,5 \mathrm{~cm}$. Les mêmes valeurs que dans l'expérience 2 ont été utilisées pour pouvoir déterminer si la présence de plusieurs directions a une influence. La progression de ces valeurs est linéaire, nous avons aussi testé une série avec progression exponentielle comme le suggère Nesbitt [7]. C'est la troisième série et donc les amplitudes sont $0,4 \mathrm{~cm}, 0,8 \mathrm{~cm}$ et $1,6 \mathrm{~cm}$.

Résultats et discussion. Les erreurs en direction sont toujours minimes : seuls deux utilisateurs ont fait une faute sur les trois séries. C'est pourquoi les valeurs ne seront pas détaillées. On peut conclure assez facilement que le fait d'utiliser plusieurs amplitudes en même temps que plusieurs directions ne perturbe pas la discrimination des directions.

Pour les erreurs d'amplitude, en ce qui concerne la première série la moitié des utilisateurs n'a pas fait de fautes et l'autre n'en a fait qu'une sur les cent cinquante impulsions. La discrimination de telles impulsions est donc claire. Par contre avec trois amplitudes c'est plus problématique. Les erreurs de discrimination d'amplitude pour la série 2 sont résumées dans la table 3. Comme pour l'expérience 2 nous avons séparé les erreurs par amplitude proposée sur chaque ligne et la dernière colonne représente le pourcentage d'erreurs sur le total de tous les utilisateurs pour l'amplitude de la ligne. On ob- 
tient $16 \%$ d'erreurs en moyenne sur toute la série. 3\% des petites impulsions $(0,4 \mathrm{~cm})$ sont mal interprétées, $19 \%$ des moyennes $(0,95 \mathrm{~cm})$ et $27 \%$ des grandes $(1,5 \mathrm{~cm})$. Si on compare ces résultats avec ceux de l'expérience 2 on peut constater qu'en moyenne il y a deux à trois fois plus d'erreurs. Il est clair que les différentes directions ont perturbé la discrimination des amplitudes. Cette constatation est corroborée avec les sentiments des utilisateurs recueillis après les tests : ils avaient l'impression de sentir des amplitudes différentes selon les directions. Les utilisateurs ont été perturbés car l'apprentissage qui leur a été proposé était très sommaire.

\begin{tabular}{|c|c|c|c|c|c|c|c|}
\hline Amplitude & \multicolumn{9}{|c|}{ Utilisateur } & Erreurs \\
$(\mathbf{c m})$ & 1 & 2 & 3 & $\mathbf{4}$ & 5 & $\mathbf{6}$ & $(\%)$ \\
\hline 0,4 & 0 & 1 & 3 & 5 & 0 & 0 & $3 \%$ \\
0,95 & 10 & 10 & 19 & 4 & 5 & 2 & $19 \%$ \\
1,5 & 9 & 17 & 20 & 18 & 13 & 9 & $27 \%$ \\
\hline Total & 19 & 28 & 42 & 27 & 4 & 11 & $16 \%$ \\
\hline
\end{tabular}

Table 3 : Expérience 3, série 2 : erreurs en amplitude

Vous pouvez consulter les erreurs de discrimination d'amplitude de la troisième série dans la table 4 . De manière générale on remarque que les erreurs sont un peu moins élevées. On a toujours 3\% des petites impulsions qui sont mal reconnues, mais maintenant on a respectivement $11 \%$ et $19 \%$ des moyennes et des grandes impulsions qui sont mal interprétées.

\begin{tabular}{|c|c|c|c|c|c|c|c|}
\hline Amplitude & \multicolumn{9}{|c|}{ Utilisateur } & Erreurs \\
$(\mathbf{c m})$ & 1 & 2 & 3 & 4 & 5 & 6 & $(\%)$ \\
\hline 0,4 & 1 & 0 & 2 & 1 & 3 & 1 & $3 \%$ \\
0,8 & 2 & 23 & 8 & 2 & 1 & 1 & $19 \%$ \\
1,6 & 7 & 24 & 13 & 5 & 3 & 1 & $27 \%$ \\
\hline Total & 1 & 47 & 23 & 8 & 7 & 3 & $16 \%$ \\
\hline
\end{tabular}

Table 3 : Expérience 3, série 3 : erreurs en amplitude

On peut constater qu'il y a un tiers d'erreurs en moins par rapport à la série précédente, seul un utilisateur s'est trompé plus de fois. Le pourcentage de petites amplitudes erronées ne change pas. En revanche il y a un quart des erreurs en moins pour l'amplitude moyenne et presque $40 \%$ pour la grande amplitude. Il semblerait donc que la progression exponentielle des amplitudes soit plus discriminatoire que la progression linéaire. Cependant les erreurs restent élevées ( $11 \%$ en moyenne). Cela nous pousse à croire que sans apprentissage la discrimination d'impulsions de trois amplitudes différentes n'est pas possible dans des conditions normales d'utilisation du périphérique utilisé.

\section{CONCLUSION}

L'affichage d'informations par des impulsions de plusieurs directions et amplitudes est possible. Les études détaillées dans cet article nous permettent d'affirmer que la discrimination de direction est aisée, ainsi que la discrimination de deux amplitudes. Cependant la discrimination d'impulsions de trois amplitudes différentes pose problème. Il serait intéressant d'étudier de nouvelles valeurs et surtout si un apprentissage approprié permet cette discrimination. Concernant la discrimination de deux amplitudes on pourrait s'intéresser à l'écart minimal des valeurs pour avoir une discrimination sans ambiguïté.

En utilisant six directions et deux amplitudes on peut utiliser le principe de codes hiérarchiques des earcons et des tactons sur deux niveaux. Nos travaux futurs sur la discrimination d'impulsion de durées différentes permettront peut être de créer un troisième niveau. Dans de prochaines études nous nous intéresserons à d'autres alphabets.

\section{REMERCIEMENTS}

Ce travail est financé par le projet européen MICOLE (IST-2003-511592) et par la CA2M (Communauté d'Agglomération de Metz Métropole) dans le cadre du projet PICOB.

\section{BIBLIOGRAPHIE}

1. Reachin API 3.2 Programmer's Guide. http://www.reachin.se

2. Stephen A. Brewster et Lorna Brown. Non-visual information display using tactons. Dans CHI'04 : Extended abstracts on Human factors in computing systems, pages 787-788, Vienne, Autriche, Avril 2004. ACM Press.

3. Stephen A. Brewster, Peter C. Wright, et Alistair D. N. Edwards. An evaluation of earcons for use in auditory human-computer interfaces. Dans CHI'93 : Proceedings of the conference on Human factors in computing systems, pages 222-227, Amsterdam, The Netherlands, Avril 1993. ACM Press.

4. William W. Gaver. Synthesizing auditory icons. Dans CHI'93 : Proceedings of the conference on Human factors in computing systems, pages 228235, Amsterdam, The Netherlands, Avril 1993. ACM Press.

5. Karon E. MacLean et Mario J. Enriquez. Perceptual design of haptic icons. Dans Proceedings of the $3^{\text {rd }}$ International Conference Eurohaptics 2003, pages 351-363, Dublin, UK, July 2003. ACM Press

6. Thomas M. Massie et J. Kenneth Salisbury. The phantom haptic interface : a device for probing virtual objects. Dans Proceedings of the ASME Winter Annual Meeting, Symposium on Haptic Interface for Virtual Environment and Teleoperator Systems, pages 295-301, Chicago, Novembre 1994. vol. 1.

7. Keith V. Nesbitt. Experimenting with haptic attributes for display of abstract data. Dans Proceedings of the $2^{\text {nd }}$ International Conference Eurohaptics 2002, pages 150-155, Edimbourg, Ecosse, Juillet 2002. ACM Press. 